\title{
Metodología de selección de procesos para la gestión de servicios en las pymes
}

\section{Extracto:}

Es un hecho que cada vez más empresas basan su operativa en las tecnologías de la información y las comunicaciones (TIC). Los modelos de gestión tradicionales necesitan ser adaptados a esta nueva realidad. Para ello están surgiendo iniciativas (COBIT [control objectives for information and related technology], CMMI [capability maturity model integration], ITIL [information technology infrastructure library], etc.) que pretenden orientar sobre los procesos, las métricas y los indicadores de gestión de la tecnología más adecuados.

En este trabajo nos centraremos en ITIL, máximo exponente de lo que se ha dado en llamar Gobierno TI. ITIL es un referente en las empresas de servicios de tecnología y en los departamentos de TIC de cualquier empresa. Ello se debe a que la organización y cobertura de los procesos propuestos para la correcta gestión de servicios informáticos aporta un grado de utilidad superior al resto de iniciativas existentes.

La implantación de un modelo de gestión basado en los procesos ITIL supone para las empresas una decisión relevante: ¿qué procesos implantar?, ¿por cuál empezar?, etc. La contestación a estas y otras preguntas no es fácil porque la adopción de estos procesos implica una inversión económica importante. Este trabajo da respuesta a la pregunta de qué orden de implantación de procesos seguir de modo que maximicemos el posicionamiento de la empresa en su sector, el posicionamiento frente a empresas de igual tamaño o cualquier otro parámetro que se defina.

Palabras clave: ITIL (information technology infrastructure library), procesos, orden, secuencia, pyme, servicios.

\footnotetext{
1 J. L. Rubio Sánchez, vicerrector universidad-empresa de la Universidad a Distancia de Madrid (UDIMA).

${ }^{2}$ C. Burgos Garcia, rectora de la Universidad a Distancia de Madrid (UDIMA).
} 


\section{Process}

\section{selection}

\section{methodology}

\section{for service}

\section{management in SME}

\section{Abstract:}

It is a fact that more and more companies operations lay in information and communication technologies (ICT). Traditional management models need to be adapted to this new reality. That is why some initiatives are emerging (COBIT [control objectives for information and related technology], CMMI [capability maturity model integration], ITIL [information technology infrastructure library], etc.) which pretend to guide about the processes, metrics and technology management indicators most suitable.

This document focuses in ITIL, that is the best representation of what has been called IT Governance. ITIL is a reference in technology services companies and in ICT departments of any company. That is due to the high level of utility provided by the organization and coverage of the processes proposed.

Implantation of a management model based in ITIL processes forces companies to a relevant decision: which processes should be implemented?, which one should be the first one?, etc. The answer to this and other questions is not easy because the adoption of these processes implies an economical investment. This article shows an approach to the implementation order so we can optimize the position of the company in front of the competence in its sector, in front of similar sized companies or any other parameter we could define.

Keywords: ITIL (information technology infrastructure library), processes, order, sequence, SME, services.

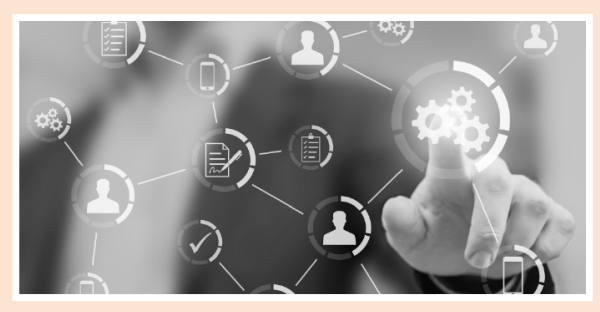

\section{INTRODUCCIÓN}

\subsection{Marco de referencia y objetivo}

El sector de las nuevas tecnologías ha permitido la creación de numerosas empresas que basan sus modelos de negocio en el uso intensivo de las TIC, así como la modernización de muchas otras que han mejorado sus procesos productivos a costa de la incorporación masiva de tecnología.

Sin embargo, la decisión de qué procesos mecanizar, automatizar o industrializar no es fácil. Si tenemos en cuenta que muchas de las empresas que basan sus modelos de negocio en el uso intensivo de la tecnología son empresas de servicios, la dificultad de valorar qué procesos seleccionar es aún mayor dada la inexistencia de modelos claros que ayuden a la toma de decisiones. En este sentido cabe indicar la ausencia generalizada de referencias acerca del retorno de la inversión por la adopción de determinada práctica 0 proceso concreto en una empresa.

\section{[...] la decisión de qué procesos} mecanizar, automatizar o industrializar no es fácil. Si tenemos en cuenta que muchas de las empresas que basan sus modelos de negocio en el uso intensivo de la tecnología son empresas de servicios, la difficultad de valorar qué procesos seleccionar es aún mayor dada la inexistencia de modelos claros que ayuden a la toma de decisiones 


\section{En los inicios de la informática}

\section{[...] las empresas fabricantes}

de hardware eran también las

creadoras del software. Este

oligopolio del mercado solo se

rompió con la aparición de lenguajes

de programación avanzados

Para comprender mejor el panorama ante el que nos encontramos, debemos tener en cuenta los siguientes aspectos:

- En los inicios de la informática, más o menos a finales de los años cincuenta del siglo XX, las empresas fabricantes de hardware eran también las creadoras del software. Este oligopolio del mercado solo se rompió con la aparición de lenguajes de programación avanzados que permitieron separar la construcción de hardware y la elaboración de los programas que sobre ellos se ejecutaban. Dicha separación dio lugar a la aparición de numerosas empresas que podían hacer programas sin ser fabricantes de hardware, es decir, comienzan a aparecer las primeras empresas de servicios en el sector informático.

Esta separación condujo a una especialización de las empresas en fabricantes de hardware y en fabricantes de software, lo cual facilitó la eclosión de las nuevas tecnologías en las décadas de los ochenta y noventa. Este auge tan desmesurado acercó

\section{[...] es importante reseñar}

la aparición de empresas de

servicios de todo tipo (call

center, telemarketing, brókeres,

agregadores de noticias, etc.)

que basan todo su negocio en la

correcta aplicación de procesos

tecnológicos y en la correcta

gestión de la tecnología de manera un tanto forzada la tecnología a muchas empresas, pues los costes de su implantación se redujeron notablemente, como explican Mas y Quesada (2005). Sin embargo, en la mayor parte de los casos, la implantación de tecnología se realizaba sin criterios claros de optimización ni desde un punto de vista económico ni técnico.

- Este mismo crecimiento de soluciones tecnológicas originó nuevos problemas hasta entonces impensables: el modo de gestionar estos grandes proyectos basados en las TIC no estaba resuelto y en muchas ocasiones dichos proyectos acababan en fracaso, por muy distintas razones. Unas veces las empresas no estaban preparadas para asumir dichos cambios tecnológicos, en otras ocasiones la tecnología no estaba lo suficientemente madura, en otras los cambios en los procesos internos de las empresas no se acometían adecuadamente, etc. Sea como fuere, se creó un caldo de cultivo que facilitó la irrupción de manuales de buenas prácticas de gestión en el ámbito tecnológico. Este escenario permanece hoy día y, aunque las propuestas son variadas, ya hay algunas prácticas y estándares que destacan por encima de otras como «más recomendables» 0 «más completas».

- Para completar el panorama es importante reseñar la aparición de empresas de servicios de todo tipo (call center, telemarketing, brókeres, agregadores de noticias, etc.) que basan todo su negocio en la correcta aplicación de procesos tecnológicos y en la correcta gestión de la tecnología.

- Tal y como explican Gorriti y Álvarez (2005), Europa no ha explotado de una forma tan positiva como EE. UU. el uso masivo de la tecnología. Esto se ve reflejado en el hecho de que la productividad no ha crecido de igual forma tras las inversiones realizadas. En dicho artículo se plantea una posible respuesta, fundamentada en que además de incorporar tecnología es necesario incorporar procesos para gestionar adecuadamente dicha tecnología.

- En general, y por lo que respecta a las empresas españolas, existe una baja preocupación por las calidad, tal y como se constata en Aragón y Rubio (2005). Este hecho, unido a los pocos ejercicios existentes en el mercado -como señalan Muñoz y Ulloa (2011)- que permitan entender fácilmente todas las normas, estándares, prácticas, normativas, guías, etc., publicados, da una idea de la distancia que hay entre lo que hacen las empresas y lo que deberían hacer. 
En definitiva, nos encontramos con un sector empresarial dispuesto a afrontar inversiones en tecnología, con el riesgo de que dichos proyectos no retornen los beneficios esperados, entre otras causas por no adoptar los procesos necesarios para ello y por no adaptar sus procesos internos de gestión de la tecnología adecuadamente.

En este sentido, una de las demandas más interesantes de las empresas, sobre todo de las pequeñas y medianas, es ser orientadas sobre el conjunto de procesos y procedimientos que más y mejor pueden posicionar a la empresa en relación a las inversiones realizadas en tecnología.

Dicho posicionamiento se puede realizar desde distintas ópticas, si bien en este trabajo nos circunscribimos a la mejora del posicionamiento respecto a las empresas de su sector y respecto a las empresas de su tamaño.

No escapa el hecho de que este planteamiento se debe enmarcar en el ámbito de los estándares, manuales, guías y normativas sobre el buen gobierno de Ias TIC, tales como COBIT, ITIL, SWEBOK, COSO y otros semejantes que presentamos en la siguiente sección. No obstante, ITIL es con diferencia el que mayor implantación y aceptación tiene en el mercado (Institute of Systems Science, 2013; Bahsani et al., 2011; Heschl, 2004), por lo que será el marco de actuación de la presente propuesta.

A partir de los puntos expuestos anteriormente estamos en condiciones de fijar el objetivo del presente trabajo:

\section{Objetivo:}

Definición de una metodología rigurosa de selección de procesos ITIL para su implementación en una pyme -tal y como se definen por el Ministerio de Industria (Ministerio de Industria de España, 2013)- de modo que se optimice el posicionamiento de dicha empresa entre las de su mismo sector y tamaño.

Es decir, el objetivo es la elaboración de un algoritmo, el cual, a partir de datos empíricos, nos ofrezca una respuesta sobre qué proceso ITIL implementar en primer lugar. Dicho algoritmo se ha de caracterizar por ser el que optimice el posicionamiento de la

\section{[...] Europa no ha explotado de una forma tan positiva como EE. UU. el uso masivo de la tecnología. Esto se ve reflejado en el hecho de que la productividad no ha crecido de igual forma tras las inversiones realizadas}

empresa en cuanto a procesos (ITIL) implementados en comparación con empresas de su mismo tamaño y con empresas de su mismo sector.

Es preciso hacer una observación. En este caso estamos planteando optimizar el posicionamiento en relación a empresas de igual tamaño y en relación a empresas de igual sector. La razón para haber seleccionado estos dos criterios se basa en la información obtenida en las bases de datos disponibles y en el hecho de que la extensión del algoritmo para el empleo de otros criterios o más criterios es inmediata, como se indica más adelante.

\subsection{Estructura y organización}

El trabajo comienza con una breve presentación de los diferentes estándares y marcos/manuales de buenas prácticas empleados en las empresas de servicios tecnológicos y en los departamentos TIC de cualquier empresa.

En segundo lugar, abordaremos la metodología de trabajo que se ha seguido para la elaboración del algoritmo de ayuda a la decisión -objeto principal de este trabajo de investigación-.

Posteriormente, y dado que el algoritmo basa su decisión en datos empíricos, se presentará la toma de datos realizada y, a continuación, se expondrá formalmente el fundamento matemático y el procedimiento para la toma de decisión sobre el proceso que se va a implementar.

Por último, se expondrán las líneas de mejora y evolución identificadas y se incluirá la bibliografía. 


\section{ESTÁNDARES, NORMATIVAS Y GUÍAS EN EL ÁMBITO DE LOS PROCESOS PARA EL GOBIERNO Y LA GESTIÓN DE LAS TIC}

En el ámbito de la gestión de tecnologías de la información dentro de las empresas, existen numerosas guías de buenas prácticas, normativas y estándares representados mediante un sinfín de siglas. En general, hay pocos trabajos dedicados a poner orden entre todas estas iniciativas. Entre estos podemos citar el de Muñoz y Ulloa (2011).

Las iniciativas más representativas las podemos clasificar en las siguientes:

- Marcos de trabajo/prácticas/metodologías. En este grupo enmarcamos el conjunto de iniciativas que indican qué y cómo hacer algo. Es decir, se identifican los procesos que se van a implementar para acometer un objetivo determinado y cómo implementarlos. Destacamos, entre otros:

- COBIT.

- ITIL.

- COSO (Committee of Sponsoring Organizations of the Treadway Commission)

- MOF (Microsoft operations framework).

- Medición y mejora de procesos. En este grupo situamos todas aquellas iniciativas que se encargan de determinar cómo medir los procesos y cómo mejorar el resultado de estos:

- CMMI.

- EFQM (European Foundation for Quality Management)

- Lean IT.

- Six Sigma.

- Estándares. Dentro de los estándares situaremos las iniciativas que persiguen certificar el cumplimiento de determinados marcos de trabajo, prácticas o metodologías. Destacan:

- ISO 20000.

- ISO 27001.

- ISO 19770.

En la figura 1 representamos de manera esquemática la finalidad de cada iniciativa:

Figura 1. Prácticas/estándares/mejora

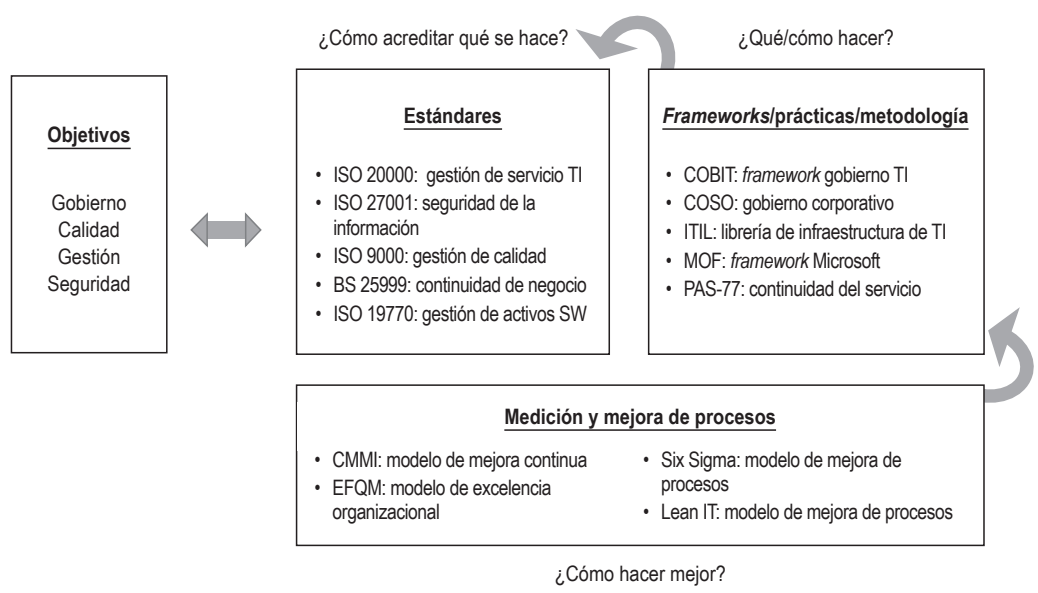

Fuente: elaboración propia. 
En general, podemos afirmar que el primer paso suele ser la identificación de una necesidad. En este caso, en las empresas tecnológicas o en los departamentos de tecnología de cualquier empresa. A partir de aquí los actores del sector (organizaciones, asociaciones, empresas, etc.) tratan de definir manuales, metodologías, guías de buenas prácticas, etc., de modo que sirvan de guía sobre qué hacer, tal y como se puede apreciar en la figura 1.

El paso inmediatamente posterior consiste fundamentalmente en abordar mejoras sobre lo indicado en las guías anteriormente definidas, normalmente estructuradas en forma de procesos.

Todo este camino concluye con la creación de un estándar que permite que las entidades certificadoras emitan los correspondientes certificados de adecuación a estándares basados en las guías y manuales previamente definidos por los actores implicados.

Dada la amplia aceptación y reconocimiento de ITIL en el mercado, como se indica en estudio del Institute of Systems Science (2013), a continuación presentaremos una breve descripción de esta guía de procesos.

\subsection{Breve introducción a ITIL}

ITIL representa un conjunto de prácticas/procesos orientados a la gestión, desarrollo y operación de las tecnologías de la información (más información en Bon et al., 2012). ITIL tiene como objetivo definir un conjunto de procesos y procedimientos para todas aquellas entidades que desarrollen servicios basados en tecnología y para aquellas que tengan un departamento de tecnología (prestador de servicios tecnológicos a otros departamentos internos). Es importante recalcar que ITIL no está pensado para un sector de empresas determinado, sino que representa un marco genérico para todo tipo de empresas.

ITIL 2011 comprende cinco grandes grupos de procesos para la gestión de los departamentos TIC o de la empresa de servicios tecnológicos, cuyos principales procesos y grupos de procesos son:

- Grupo de procesos de estrategia del servicio. En este grupo encontramos todos aquellos que pretenden garantizar la viabilidad de los servicios. Inciden en los aspectos financieros, de oferta y demanda de servicios, garantizando la coherencia entre ellos. Los procesos más relevantes que se identifican son:

- Gestión Financiera.

- Gestión del Portfolio.

- Gestión de la Demanda.

- Gestión de la Estrategia.

- Gestión de las Relaciones del Negocio.

- Grupo de procesos de diseño del servicio. Este grupo trata de dar respuesta a cómo prestar el servicio, incidiendo en el modelo del servicio prestado en función de los recursos, garantizando su prestación y definiendo procesos de recuperación ante caídas, catástrofes, etc. Los procesos que se identifican son:

- Gestión del Catálogo de Servicios.

- Gestión de Niveles de Servicio.

- Gestión de la Disponibilidad.

- Gestión de la Capacidad.

- Gestión de la Continuidad de los Servicios de TI.

- Gestión de Proveedores.

- Gestión de la Seguridad de Información.

- Coordinación del Diseño.

- Grupo de procesos de transición del servicio. Comprende el grupo de procesos encargados de asegurar que el paso desde el diseño hasta la prestación propiamente dicha de un servicio por una empresa o departamento se realiza de forma segura. Esto implica la identificación de procedimientos de roll-back, identificación de alternativas para prestar el servicio por la empresa si hubiera incidentes, aseguramiento de que todos los recursos están disponibles, etc. Los procesos que se identifican son:

- Gestión de la Configuración y Activos.

- Gestión del Cambio.

- Gestión del Conocimiento.

- Planificación y Apoyo a la Transición.

- Gestión de Release y Despliegue.

- Gestión Validación y Pruebas.

- Evaluación.

- Grupo de procesos de operación del servicio. La operación del servicio se ocupa del seguimiento del servicio, registrando eventos, incidentes, problemas, accesos y, en general, todo lo que afecta a la prestación del servicio por parte del 
departamento TIC o la empresa de servicios. Los principales procesos que identifican son:

- Gestión de Incidencias.

- Gestión de Problemas.

- Cumplimiento de Solicitudes.

- Gestión de Eventos.

- Gestión de Accesos.
- Grupo de procesos de mejora del servicio. Los procesos de mejora del servicio están orientados a mejorar los índices de calidad del servicio prestado. El proceso que se identifica es:

- Proceso de Mejora (continuum service improvement).

\section{- Actividades específicas dentro de cada grupo de servicio.}

\section{METODOLOGÍA DE TRABAJO}

Una vez definido el problema que se pretende resolver y realizada una breve introducción a ITIL, pasamos a exponer la metodología de trabajo seguida, lo cual ayudará en la comprensión de la solución planteada.

\subsection{Obtención de datos}

Para poder diseñar y posteriormente emplear el algoritmo de selección de procesos que se van a implementar, antes que cualquier otra actividad, ha sido necesario construir una base de datos con información proveniente de empresas ${ }^{3}$. Para ejecutar este punto se ha realizado una encuesta en la que se han registrado datos sobre el tamaño, el sector y la antigüedad de cada empresa, el número de empleados que están dedicados a tareas tecnológicas, conocimiento sobre procesos de gestión TIC, etc., y en la que se preguntó, además, acerca del grado de implementación de cada uno de los procesos y sobre el plazo de implementación de dichos procesos -si lo hubiera-.

En la tabla 1 figuran los datos técnicos de la encuesta que se ha realizado.

\section{Tabla 1. Características de la encuesta}

\begin{tabular}{|c|c|}
\hline 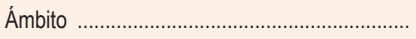 & Comunidad de Madrid. \\
\hline 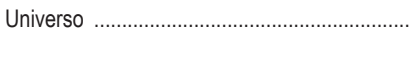 & $\begin{array}{l}\text { Empresas de } 1 \text { a } 250 \text { empleados con sede social en la Comunidad de Madrid y de } \\
\text { cualquier sector (se excluyen sociedades unipersonales). }\end{array}$ \\
\hline Tipo de encuesta .......................................... & Cuestionario web remitido por e-mail con recepción automática de respuestas. \\
\hline Formato de encuesta & Web. \\
\hline 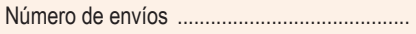 & 150 \\
\hline Número de respuestas mínimo requerido ....... & 60 \\
\hline Número de respuestas obtenido ....................... & 64 \\
\hline Nivel de confianza ........................................... & $90 \%$ \\
\hline Error de muestreo & $<8,5 \%$ \\
\hline 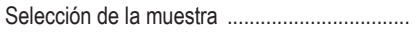 & Aleatoria y monoetapa \\
\hline
\end{tabular}

\footnotetext{
${ }^{3}$ Se han considerado pymes de la Comunidad Autónoma de Madrid con más de un empleado como representativas de las pymes.
} 
Las respuestas obtenidas en esta encuesta se emplearán como fuente de datos para los cálculos que realice el algoritmo de selección de procesos. Esto significa que el procedimiento o algoritmo de selección de procesos que se va a implementar obtendrá sus resultados en función de la información recopilada en esta encuesta, de modo y manera que cuantas más empresas utilicen la herramienta de selección diseñada, mayor será la riqueza informativa de la base de datos empleada, ya que los datos de cada empresa que lo utiliza son incorporados a la base de conocimiento disponible.

\subsection{Diseño del procedimiento de selección}

El siguiente paso consiste en la definición y el diseño de un algoritmo o procedimiento de cálculo que, a partir de los datos obtenidos en las encuestas y de las características de una empresa concreta, permita identificar qué procesos debe implementar en primer lugar dicha empresa. Es necesario disponer de la base de datos anterior, puesto que el modelo de decisión es un modelo comparativo que tiene como objeto mejorar el posicionamiento de la empresa entre aquellas de su sector, tamaño, antigüedad, etc.

Como se ha indicado anteriormente, cuanto mayor sea el número de empresas que emplean el algoritmo, mayor riqueza de información tendrá la base de datos y, por tanto, más útil será la respuesta que obtengamos del algoritmo.

La definición o el diseño del algoritmo representa la parte central del trabajo, puesto que es el elemento nuclear de la decisión que se proponga a la empresa a la hora de seleccionar los procesos que se van a implementar. La metodología empleada en la exposición es la siguiente:

- Se han establecido las bases teórico-matemáticas del algoritmo de optimización de la selección de procesos.

- Se ha definido una metodología o procedimiento para el uso del algoritmo de selección de los procesos ITIL que se van aimplementar.

Es por ello que este punto será ampliamente desarrollado en los siguientes apartados.

\section{La definición en sí misma del algoritmo para la toma de decisiones no representa una utilidad para las pymes si no va acompañada de una herramienta que permita su utilización de manera cómoda}

\subsection{Implementación}

La definición en sí misma del algoritmo para la toma de decisiones no representa una utilidad para las pymes si no va acompañada de una herramienta que permita su utilización de manera cómoda. Es por ello que, sin ser el aspecto sobre el que se pretenda incidir, resulta necesario mencionar el desarrollo e implementación de dicho algoritmo en hojas de cálculo para su utilización de forma sencilla con herramientas ofimáticas.

Si bien, desde un punto de vista técnico, hubiera sido mejor solución adoptar un lenguaje de programación de propósito general, se ha optado por la solución de implementar el algoritmo mediante hojas de cálculo, puesto que ello permitirá la utilización por las pymes de una forma sencilla.

No obstante, tal y como se ha indicado, la presentación exhaustiva de la herramienta desarrollada para la aplicación del procedimiento que se va a exponer a continuación excede el objetivo de este trabajo.

\subsection{Validación}

El último paso consiste en la prueba y evaluación del algoritmo implementado en la suite ofimática con el fin de valorar sus resultados y obtener una realimentación de las empresas en las que se ha hecho uso del algoritmo de selección.

La interface de usuario de la herramienta desarrollada con la implementación del algoritmo es extremadamente sencilla y ha permitido a pymes comenzar a utilizar empíricamente el algoritmo para la toma de decisiones sobre los procesos.

En cualquier caso, la presentación de los resultados empíricos a raíz de la utilización del algoritmo excedería el alcance del trabajo y, por ello, no están incluidos entre los objetivos del mismo. 


\section{DEFINICIÓN DEL PROCEDIMIENTO ALGORÍTMICO DE SELEC- CIÓN DE PROCESOS}

\subsection{Definiciones previas}

Para la exposición de este punto es importante tener presente que el problema que se pretende resolver es el de definir un algoritmo de selección de cuál es el proceso -de los descritos en ITIL- que una pyme debe implementar en primer lugar con el objeto de minimizar la distancia a sus competidores.

De la encuesta realizada, y aunque dicho estudio no se incluye por acotar el trabajo, se concluye que, de las características disponibles de cada empresa, las que más afectan en los procesos que estas tienen implementados son el tamaño y el sector en el que operan. En contraposición a esto, otros factores como la antigüedad resultan poco relevantes o determinantes para que la empresa tenga más o menos implementados los procesos de gestión tecnológicos analizados.

Por ello, y sin que ello represente una pérdida de generalidad en el estudio, pues la generalización a $n$ criterios es realmente sencilla, definiremos el algoritmo de selección teniendo en cuenta dos criterios de decisión:

- Tamaño de la empresa.

- Sector de la empresa.

Es decir, el criterio para seleccionar qué proceso tecnológico implementar en primer lugar dependerá del tamaño, y en segundo lugar, del sector en el que opera la empresa, o dicho de otra forma, del posicionamiento de la pyme respecto a las empresas de su sector $y$ respecto a las empresas de su tamaño.

La idea que perseguimos es la de definir una métrica o parámetro que nos de una idea de la situación de la pyme (en cuanto a implementación de procesos tecnológicos se refiere) respecto a empresas de igual tamaño y/o de igual sector. Este parámetro debe contener información sobre todos y cada uno de los potenciales procesos que se van a implementar y debe permitirnos tomar una decisión sobre la idoneidad de implementar uno u otro.

\section{Definimos el grado de implementación}

Antes de comenzar la exposición teórica del algoritmo es importante resaltar que en la encuesta para la obtención de los datos se preguntaba a las empresas por la implementación de cada proceso y estas podían responder:

- Que el proceso no estaba implementado y que no tenían previsión de tenerlo implementado a corto plazo.

- Que el proceso no estaba implementado, pero que a medio/largo plazo tenían previsto implementarlo.

- Que el proceso estaba implementado o que a corto plazo lo estaría.

Por ejemplo, al ser cuestionadas por el proceso de Gestión de Incidencias, algunas empresas indicaban que no tenían implementada una gestión de incidencias en su operativa y que no tenían previsto implementarla, mientras que otras ya lo tenían implementado o tenían previsto hacerlo a medio o largo plazo. Esto se realizó con todas las empresas, para todos y cada uno de los $P$ procesos indicados en el apartado 2.1 .

Denotemos por $E$ una empresa cualquiera a la que queremos indicar qué proceso debe implementar en primer lugar. Sea $P_{i}$ un proceso cualquiera de los $P$ potenciales procesos. Sea $d_{i}$ la valoración del proceso $P_{i}$ en la empresa $E$. Los posibles valores de $d_{i}$ son:

(1)

$d_{i}(E)=\left\{\begin{array}{l}1 \text { si } P_{i} \text { no está implementando y no está planificado } \\ 2 \text { si } P_{i} \text { está planificado a medio/largo plazo } \\ 3 \text { si } P_{i} \text { está implementando o planificado a corto plazo }\end{array}\right\}$

Por ejemplo, si $E$ es la empresa Formación SL, que tiene implementado el proceso de Gestión de Incidencias, será:

$$
d_{19}(E)=3
$$

puesto que el proceso $P_{19}=$ Gestión de incidencias. 
Mientras que si dicha empresa $E$ no tiene implementado el proceso $P_{3}=$ Gestión de la Demanda, será:

$$
d_{3}(E)=1
$$

Observemos que la definición dada por la expresión (1) del parámetro $d_{i}$ coincide con las respuestas que se ofrecian en la encuesta. Es fácil observar que la mejor puntuación que una empresa puede obtener en un proceso $P_{i}$ es 3 . El hecho de que solo se ofrezcan tres respuestas posibles en la encuesta no resta generalidad, puesto que, en caso de existir más opciones en la respuesta, bastaría redefinir la expresión (1) e incorporar el resto de respuestas.

En todo caso, y para la presentación del algoritmo, seguiremos con el caso particular de $M=3$, es decir, tres respuestas posibles a las preguntas de la encuesta.

\section{Definimos el sector empresarial}

Denotamos como $S(E)$ el sector al cual pertenece la empresa $E$, de los $j=16$ sectores $^{4}$ posibles, y lo expresamos como:

$$
S(E)=s_{j}
$$

En la encuesta realizada, las empresas se clasificaron atendiendo a estos 16 sectores de actividad, de modo que:

$$
S(E)=s_{j} \mid s_{j} \in\left\{\begin{array}{l}
s_{1}=\text { Sector TI } \\
s_{2}=\text { Sector alimentación } \\
\cdots \\
\cdots \\
s_{16}=\text { Otros }
\end{array}\right\}
$$

Así, por ejemplo, diremos que para una empresa $E=$ = Formación SL:

$$
S(E)=s_{4}
$$

pues $s_{4}$ se corresponde con el sector «Formación».

\section{Grado de implantación de los procesos en cada sector}

A continuación definimos $s_{i j}$ como la media de la valoración del proceso $P_{i}$ para las empresas del sector $s_{j}$ :

$$
s_{i j}=\bar{d}_{i} \forall E_{k} \mid S\left(E_{k}\right)=s_{j}
$$

Por ejemplo, el proceso Gestión de Incidencias en el sector de formación tiene un grado medio de implantación dado por:

$$
s_{19,4}=\bar{d}_{19} \forall E_{k} \mid S\left(E_{k}\right)=s_{4}
$$

En concreto, con los datos obtenidos en la encuesta, se verifica:

$$
s_{19,4}=\bar{d}_{19}=2,16
$$

\section{Grado de implantación de los procesos según el tamaño de la empresa}

Sea asimismo $t_{j}$ el tamaño de una empresa genérica E. De modo que:

$$
T(E)=t_{j}
$$

En la categorización de empresas se han definido tres clases posibles:

$$
T(E)=t_{j} \mid t_{j} \in\left\{\begin{array}{l}
t_{1}=[1 . .9] \text { empleados } \\
t_{2}=[10 . .49] \text { empleados } \\
t_{3}=[50 . .250] \text { empleados }
\end{array}\right\}
$$

También definimos $t_{i j}$ como la media de la valoración del proceso $P_{i}$ para las empresas del tamaño $t_{j}$

$$
t_{i j}=\bar{d}_{i} \forall E_{k} \mid T\left(E_{k}\right)=t_{j}
$$

donde [t] representa el número de empresas con tamaño $t_{j}$ y $E_{k}$ es cada una de las $\left[t_{j}\right]$ empresas de tamaño $t_{j}$.

\footnotetext{
${ }^{4}$ Identificación de los sectores de las empresas encuestadas:

$S_{1}=$ Servicios IT .

$S_{2}=$ Telecomunicaciones.

$S_{3}=$ Electrónica

$S_{4}=$ Educación.

$S_{5}=$ Salud y servicios sociales .

$S_{6}=$ Energía, agua, electricidad.

$S_{7}=$ Transporte, logística

$S_{8}=$ Finanzas, seguros

$S_{9}=$ Servicio legal, fiscal.

$S_{10}=$ Marketing, publicidad

$S_{11}=$ Construcción

$S_{12}=$ Alimentación.

$S_{13}=$ Inmobiliario.

$S_{14}=$ Comercio.

$S_{15}=$ Industria

$S_{16}=$ Otros.
} 
Continuando con el ejemplo, si la empresa $E=$ Formación SL tiene 8 empleados:

$$
T(E)=t_{1}
$$

$Y$ a partir de los datos recopilados en la encuesta, podríamos ver que para el proceso $P_{19}=$ Gestión de Inci- dencias en las empresas de tamaño $t_{1}$-es decir, de 1 a 9 empleados- se verifica:

$$
t_{19,1}=1,67
$$

Esto significa que las empresas de 1 a 9 empleados apenas tienen implementado el proceso Gestión de Incidencias, pues la media tiene un valor inferior a 2.

En la figura 2 podemos ver gráficamente los valores $d_{i}, s_{i j}$ y $t_{i j}$ correspondientes a la valoración del proceso $i$ en una empresa genérica $E_{k}$. Evidentemente, todos los valores de $d_{i j}$, $s_{i j}$ y $t_{i j}$ están comprendidos en el intervalo [1..3].

Figura 2. Valoración de un proceso en una empresa, sector y tamaño

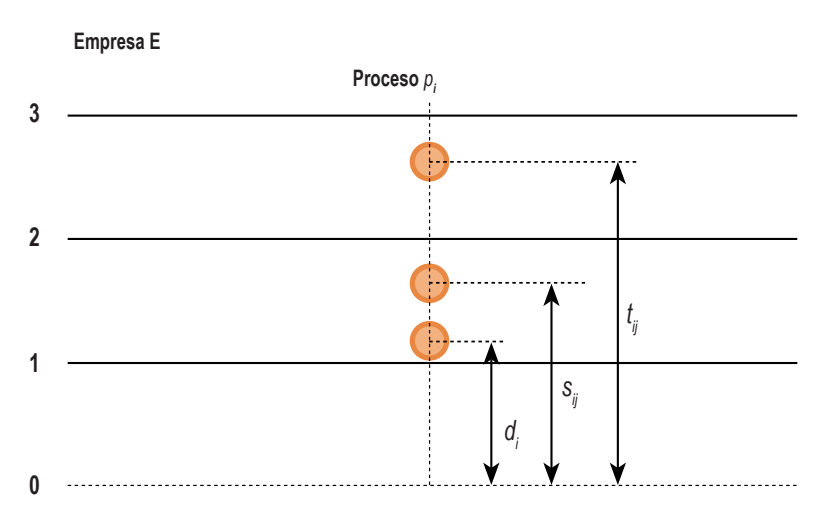

Fuente: elaboración propia.

\subsection{Definición del algoritmo de selección de procesos}

Llegados a este punto estamos ya en condiciones de plantear el algoritmo de selección de procesos que permitirá a las pymes decidir acerca de qué proceso implementar en primer lugar.

A partir de los conceptos anteriores nos interesa definir un parámetro que nos ayude a la selección del proceso que se va a implementar. Dicho parámetro debe contener información sobre la implementación de dicho proceso en las empresas del mismo sector y sobre la implementación de dicho proceso en las empresas del mismo tamaño.
Para ello, y a partir de las definiciones anteriores, fijemos los siguientes parámetros (el único punto a tener en cuenta es que 3 representa la mejor opción y 1 la peor):

$$
\begin{aligned}
& S_{i j}=\left(3-s_{i j}\right) \\
& T_{i j}=\left(3-t_{i j}\right) \\
& D_{i}=\left(3-d_{i}\right)
\end{aligned}
$$

La generalización al caso de $M$ respuestas posibles es inmediata, aunque, tal y como hemos venido indicando, el algoritmo para el caso $M=3$ no representa ninguna pérdida de generalidad en su aplicación. 
Gráficamente, podemos observar como $D_{i}, S_{i j}$ y $T_{i j}$ representan, para un proceso concreto $P_{i}$, la distancia al máximo valor (en nuestro caso 3 ) desde la respuesta de la empresa en la encuesta, la distancia al máximo valor desde la media del sector y la distancia al máximo valor desde la media de las empresas de igual tamaño:

\section{Figura 3. Distancias al máximo valor}

Empresa $\mathrm{E}$

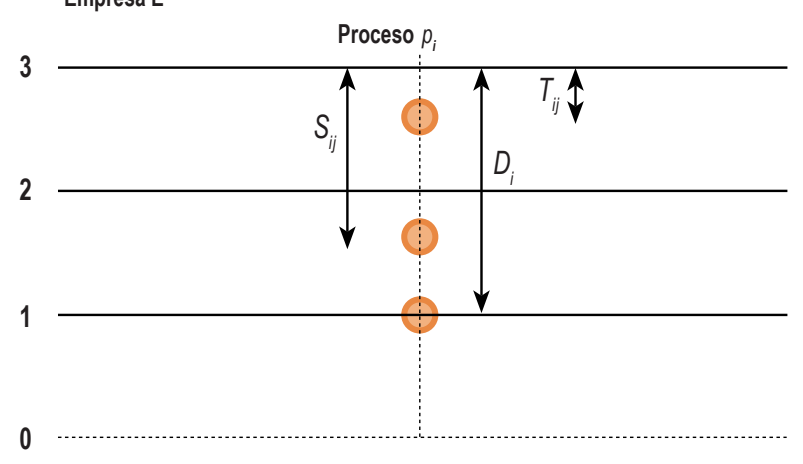

Fuente: elaboración propia.

\section{Definimos un indicador que agrupe todas las medidas}

Buscamos ahora definir un parámetro que tome valores altos cuando la empresa en cuestión está en una posición relativa mejor que las empresas de su sector y de su tamaño -de forma global- y que tome valores bajos cuando la empresa esté en una posición relativa peor que las empresas de su sector y de su tamaño.

Proponemos el siguiente indicador:

$$
F=\frac{S^{2} T^{2}}{D^{2}}
$$

que particularizado para el proceso $P_{i}$, para el sector $s_{j}$ y para las empresas de tamaño $t_{k}$ resulta:

$$
F_{i}=\frac{S_{i j}^{2} T_{i k}^{2}}{D_{i}^{2}}
$$

donde $F_{i}$ representa la evaluación del parámetro $F$ para la empresa $E$ respecto al proceso $P_{i}$.
Observemos la expresión (12.1): en dicha expresión valores bajos de $D_{i}$ indican que el proceso $i$ está implementado o en vías de estarlo próximamente en la empresa, mientras que valores altos indican que el proceso no está implementado ni va a estarlo en breve. Por otra parte, valores altos de $S_{i j}$ y $T_{i k}$ indican que el proceso $i$ no está implementado en las empresas del sector o del mismo tamaño, mientras que valores bajos indicarán que dicho proceso está implementado en las empresas del mismo sector o tamaño.

Como consecuencia de ello, el hecho de que un proceso $i$ esté implementado en las empresas del sector y/o del mismo tamaño, unido al hecho de que dicho proceso no esté implementado en la empresa en cuestión (es decir, la empresa está relativamente peor), hacen que el parámetro $F$ tome valores bajos; mientras que, al contrario, si una empresa tiene implementado el proceso i y las empresas de su sector y/o tamaño no lo tienen (es decir, la posición relativa de la empresa 
es mejor), $F$ tomará un valor muy alto. Resulta fácil deducir que el parámetro $F$ es una medida del posicionamiento relativo de una empresa para un proceso particular respecto a las empresas de su sector y/o tamaño, el cual toma valores altos para posicionamientos relativos mejores que el resto de empresas y que toma valores bajos (aunque siempre positivos) para posicionamientos relativos peores que el resto de empresas.

Por tanto, podemos formalizar:

$$
F_{i} \equiv \text { Posicionamiento_relativo } \_P_{i}\left(E_{k}\right)
$$

Esto es, $F_{i}$ representa el posicionamiento relativo de la empresa $E_{k}$ para el proceso $P_{i}$ respecto a las empresas de igual sector y/o tamaño. Como se ha indicado, en este caso se han empleado dos criterios: tamaño y sector, si bien la generalización para que $F$ tenga en cuenta más criterios es inmediata.

Una vez tenemos definido el parámetro decisor Posicionamiento_relativo, podemos plantear el criterio de selección de procesos que se van a implementar:

$$
P_{\text {opt }}=p \mid F_{p}=\min \left(F_{i}\right) \quad \forall i=\left\{1,2, . . \text { num }_{\text {proctTLL }}\right\}
$$

La expresión anterior nos indica que el proceso óptimo para ser implementado, es decir, el que más mejorará el posicionamiento de la empresa en el mercado, es precisamente aquel que en la actualidad presenta un valor menor de $F_{p}$, es decir, ofrece en la actualidad un valor de Posicionamiento_relativo más bajo.

\section{Mejoras del indicador}

No obstante, este criterio requiere una pequeña corrección. Dado que el Posicionamiento_relativo $F$ tiene en cuenta el estado de la propia empresa y el estado de las empresas del sector y del mismo tamaño, pudiera darse el caso de que el óptimo resultara un proceso que ya está implementado (es decir $D_{i}=0$ ) en la empresa. Para evitar este caso particular, debemos restringir el resultado de modo que la expresión (13) solo tenga en cuenta procesos que no estuvieran ya implementados en la empresa:

$$
P_{\text {opt }}=p \mid F_{p}=\min \left(F_{i}\right) \quad \forall i=\left\{1,2, . . \text { num }{ }_{\text {proct TLL }}\right\} \mid D_{i} \neq 0
$$

Como ejemplo de cálculo de $F_{i}$, evaluemos $F_{4}$ para la empresa $E=$ Formación, SL citada anteriormente. La empresa, con menos de 9 empleados y del sector 4 (en el caso de la encuesta, «Educación») no tiene implantado ni planificado el proceso $P_{4}=$ Gestión del Catálogo de Servicios, en cuyo caso $d_{1}=1$ (esto es, en la encuesta, la empresa responde "El proceso no está implementado y no lo estará a medio plazo»). Para las empresas de su sector y de su tamaño, a partir de la base de datos elaborada con las encuestas se obtiene $s_{1 j} \approx 1,67$ y $d_{1 j} \approx 1,56$. Por tanto, el parámetro $F_{i}$ sería:

$$
F_{1}=\frac{S_{14}^{2} T_{11}^{2}}{D_{1}^{2}}=\frac{(3-1,67)^{2}(3-1,56)^{2}}{(3-1)^{2}}
$$

El resultado de esta expresión lo tenemos en (15):

$$
F_{1}=\frac{S_{14}^{2} T_{11}^{2}}{D_{1}^{2}}=\frac{(1,33)^{2}(1,44)^{2}}{(2)^{2}}=0,91
$$

Realizando esto para todos los procesos evaluados en la encuesta tendríamos una colección de parámetros $\left(F_{1}, F_{2}, \ldots, F_{25}\right)$. Tomando el mínimo de todos ellos, obtendríamos el proceso por el cual comenzar la implantación.

\subsection{Procedimiento de selección de los procesos tecnológicos que se van a implementar}

Una vez establecida la base matemática, pasamos a exponer los pasos del procedimiento para la selección de procesos ITIL que se van a implementar:
Paso 1. Toma de datos de la empresa

El primer paso del procedimiento consiste en la toma de datos. En concreto se requiere obtener los datos 
de tamaño, sector y grado de implementación de cada uno de los 25 procesos ITIL.

Ello nos da lugar al conjunto de respuestas $d_{i}$ con valores $[1,2,3]$ para cada uno de los procesos, representando:

[no_implementado_o_largo_plazo; medio_plazo; implementado_o_corto_plazo]

Dicho conjunto de respuestas los denotamos como:

$$
\Omega_{\text {resp }}=\left\{d_{1}, d_{2}, d_{3}, \ldots, d_{i}, \ldots, d_{\text {NumProct TLL }}\right\}
$$

Paso 2. Acotar el conjunto de procesos seleccionables

Se define el conjunto $\Omega_{\text {des }}$ de procesos descartados, formado por todos aquellos procesos ya implementados (es decir, formado por los procesos que verifican $\left.D_{i}=0\right)$ :

$$
\Omega_{\text {des }}=\left\{P_{a}, P_{\beta}, P_{\gamma}, \ldots, P_{i}, \ldots, P_{d e s}\right\} \mid D_{i}=0
$$

Se define el conjunto $\Omega_{\text {rech }}$ de procesos rechazados, por todos aquellos procesos no implementables porque la empresa ha decidido posponer o rechazar su implementación $n^{5}$ :

$$
\begin{aligned}
\Omega_{\text {rech }}= & \left\{P_{\alpha}, P_{\beta}, P_{\gamma}, \ldots, P_{i}, \ldots, P_{\text {rech }}\right\} \mid \\
& \mid P_{i} \text { es rechazado }
\end{aligned}
$$

Se define el conjunto de procesos potenciales o susceptibles de ser implementados y lo denotamos con $\Omega_{\text {pot }}$ Inicialmente este conjunto está formado por todos los procesos no descartados ni rechazados aún en la empresa o en el departamento TIC:

$$
\begin{aligned}
\Omega_{\text {pot }}= & \left\{P_{1}, P_{2}, P_{3}, \ldots, P_{i}, \ldots, P_{\text {NumProclTL }}\right\} \mid \\
& \mid P_{i} \notin \Omega_{\text {des }} \wedge P_{i} \notin \Omega_{\text {rech }}
\end{aligned}
$$

El número de procesos potenciales vendrá dado por la cardinalidad de este conjunto:

$$
n_{\text {pot }}=n\left(\Omega_{\text {pot }}\right)
$$

\section{El Posicionamiento_relativo $\boldsymbol{F}$ tiene en cuenta el estado de la propia empresa y el estado de las empresas del sector y del mismo tamaño}

Paso 3. Evaluación del indicador Posicionamiento_relativo para cada proceso

Evaluación del parámetro Poscionamiento_relativo para cada uno de los $n_{\text {pot }}$ procesos $P_{i}$ contenidos en el conjunto de procesos potenciales $\Omega_{\text {pot }}$

El resultado de esta evaluación será una colección $\Omega_{\text {pos }}$ de $n_{\text {pot }}$ valores $F_{i}$ correspondientes al posicionamiento relativo de la empresa respecto a las de igual tamaño y/o sector por cada proceso evaluado:

$$
\Omega_{\text {pos }}=\left\{F_{a}, F_{\beta}, F_{\gamma}, \ldots, F_{i}, \ldots, F_{n_{p o t}}\right\} \mid P_{i} \in \Omega_{\text {pot }}
$$

Paso 4. Selección del proceso que optimiza el posicionamiento relativo de la empresa

Una vez evaluados todos los $F_{i}$, se toma el proceso $p$ que satisface la expresión (13), es decir, aquel que, formando parte de los procesos posibles en las condiciones actuales, presenta un Poscionamiento_relativo menor:

$$
P_{\text {opt }}=p\left|F_{p}=\min \left(F_{i}\right)\right| F_{i} \in \Omega_{\text {pos }}
$$

Paso 5. Reajustar el conjunto de procesos implementables

Si la empresa decide implementar el proceso $P_{\text {opt" }}$ reajustar el conjunto $\Omega_{\text {des: }}$ :

$$
\Omega_{\text {des }}=\Omega_{\text {des }} \cup\left\{P_{\text {opt }}\right\}
$$

\footnotetext{
${ }^{5}$ Ello puede deberse a criterios estratégicos, de coste, de urgencia, etc.
} 
Si la empresa decide rechazar la implementación de $P_{\text {opt }}$, reajustar el conjunto $\Omega_{\text {rech }}$ :

$$
\Omega_{\text {rech }}=\Omega_{\text {rech }} \cup\left\{P_{\text {opt }}\right\}
$$

En cualquiera de los dos casos, reajustar el conjunto de procesos potenciales:

$$
\begin{aligned}
\Omega_{\text {pot }}= & \left\{P_{1}, P_{2}, P_{3}, \ldots, P_{i}, \ldots, P_{\text {NumProcitl }}\right\} \mid \\
& \mid P_{i} \notin \Omega_{\text {des }} \wedge P_{i} \notin \Omega_{\text {rech }}
\end{aligned}
$$

El número de procesos potenciales se habrá decrementado en una unidad y vendrá dado por la cardinalidad de este nuevo conjunto:

$$
n_{\text {pot }}=n\left(\Omega_{\text {pot }}\right)
$$

Paso 6. Continuar con la selección de procesos que se van a implementar

Si se verifica:

$$
n_{\text {pot }}>0
$$

se debe volver al paso 4 con los conjuntos $\Omega$ ya reajustados.

Si no, ir al paso 7.

Paso 7. Finalización

Puesto que $n_{\text {pot }}=0$, no quedan procesos por seleccionar y el algoritmo de selección concluye.

\section{CONCLUSIONES Y LÍNEAS FUTURAS}

\subsection{Conclusiones}

Las conclusiones más relevantes del presente trabajo las podemos resumir en los siguientes puntos:

- El auge de las TIC como facilitador de las actividades de la empresa en las últimas décadas ha dado lugar a empresas con una fuerte dependencia de la tecnología ya sea porque el negocio está ligado a las TIC o porque el departamento de informática tiene una gran relevancia en la evolución del negocio. Esto ha dado lugar a una nueva necesidad, consistente en la ordenación de los procesos y actividades TIC: es lo que se ha dado en llamar «el buen gobierno de las TIC»).

- Derivada de esta necesidad están surgiendo respuestas que intentan solucionar el problema de la correcta gestión de los servicios informáticos de la empresa. Entre tales iniciativas hemos indicado ITIL -las referencias indican que es la más extendida-, si bien hay otras muy implantadas en el mercado, como COBIT y COSO.

- En general, dichas iniciativas describen qué hay que hacer para una correcta gestión de las TIC en las empresas y de hecho se reconoce y valora positivamente su aportación, pero no hay referencias sobre cómo implantar los procesos que se recomiendan. Una de las carencias más notables que encontramos hace referencia a la selección del proceso(s) que se van a implementar.

- Este hecho afecta especialmente a las pymes, que cuentan con menos recursos para realizar estudios sobre la conveniencia de implementar uno $u$ otro proceso en pos del buen gobierno de sus áreas TIC.

- El algoritmo y el procedimiento de aplicación de procesos ITIL propuesto trata de resolver este problema. Para ello se tienen en cuenta los datos recogidos en la encuesta realizada y se plantea una solución basada en la optimización del posicionamiento relativo de la empresa para cada uno de los procesos propuestos. Dicha optimización se realiza por comparación con las empresas de igual sector y/o tamaño. La extensión del algoritmo para incluir otros elementos de comparación como -por ejemplo- tamaño del departamento TIC o antigüedad de la empresa es sencilla. Asimismo, la extensión del algoritmo para soportar más posibilidades en las respuestas esinmediata.

- A partir de la formulación matemática de la solución anterior, se propone una metodología en seis pasos que da respuesta a las necesidades de las pymes a la hora de implementar los procesos ITIL. La metodología aplica de forma recurrente e iterativa el algoritmo expuesto. 


\subsection{Líneas de trabajo futuras}

El presente estudio se enmarca en un trabajo más amplio de elaboración de una metodología completa sobre implementación de procesos para la correcta gestión y gobierno TIC en una pyme. Hemos presentado el algoritmo y el procedimiento para la selección de procesos mediante la optimización del posicionamiento relativo de la empresa, en la que los criterios de decisión han sido el tamaño de la organización y el sector de la misma.

A partir de esta idea, las líneas de trabajo abiertas y en las que es necesario aportar soluciones son las siguientes:

- Es importante que el algoritmo incorpore más criterios de decisión con el fin de que la recomendación sea lo más acorde posible a las necesidades de la empresa. A título enumerativo, pero no limitativo, se proponen:

- Tamaño del departamento de tecnología. Es muy probable que uno de los factores que más afecten a las posibilidades reales de implementación de procesos tenga que ver con la cantidad de recursos que deben realizar dicha implementación.

- Tipología de los recursos TIC. Uno de los aspectos que afecta y no está estudiado es el hecho de que los recursos sean internos o estén externalizados. Se ha observado (aunque no se ha realizado un estudio riguroso) que -contrariamente a lo que podría parecer- la externalización de servicios y recursos es un elemento facilitador de la implementación del buen gobierno. Las razones no están claras, pero todo apunta a dos factores: por una parte, la menor resistencia al cambio al incorporar prácticas de buen gobierno $0^{6}$ y por otra, mayor especialización de los recursos afectados.

- Retorno de la inversión. Este elemento pasa por ser uno de lo más decisivos. La introducción en el algoritmo de algún elemento que tenga en cuenta no solo aspectos referidos a la competencia, sino factores estrictamente económicos en términos de coste y retorno esperado de la inversión mejorará la fiabilidad del proceso de toma de decisión.

- Mejoras en la formulación del algoritmo. En la formulación matemática no se ha tenido presente el hecho de que no todos los criterios (tamaño, sector, etc.) influyen por igual. Es posible pensar que el peso de todos estos criterios no sea el mismo en la decisión final y ello debería ser incorporado en el algoritmo e incluso reformular el algoritmo para que los pesos de los criterios varíen con el sector y el tamaño de la empresa sobre la que se aplica.

- Validación del procedimiento. El algoritmo, según está planteado, ofrece la mejor solución para posicionar la empresa respecto a las de su tamaño y sector. Sin embargo, resulta necesario estudiar comparativamente esta solución con otras, puesto que, aunque esta es una solución factible y de interés para las empresas ${ }^{7}$, puede no ser la que mejor represente los intereses de la pyme. Es decir, puede suceder que el interés de la pyme sea mejorar la satisfacción del cliente, minimizar los tiempos de respuesta o mejorar la calidad del producto, entre otros intereses. El algoritmo de decisión se puede modificar para que relacione los procesos disponibles con este tipo de intereses, más cercanos al negocio, y que la optimización se realice en base a estos.

- Mejora en la precisión de los datos. Este trabajo se ha realizado con una encuesta en la que se ofrecían tres posibles niveles de respuesta y se han obtenido datos que sitúan el error en el 8,5\%.

- La ampliación de la escala hasta cinco niveles puede mejorar la calidad de la respuesta y sin necesidad de modificar sustancialmente el algoritmo ni el procedimiento de toma de decisión.

- La incorporación de más empresas en la base de datos redundará en una mayor precisión de las repuestas obtenidas del procedimiento. Aunque la encuesta se ha implementado en una herramienta disponible a través de internet de modo que las empresas pudieran dejar los datos fácilmente, es preciso incidir en la necesidad de incrementar los datos disponibles para una mejora de la respuesta.

- Otras mejoras en la toma de datos de la encuesta. La toma de datos se puede mejorar incorporando datos de otros sectores, estratificando las muestras o realizando tomas multietapa.

\footnotetext{
${ }^{6}$ Por ejemplo, las propuestas por ITIL o por COBIT.

${ }^{7}$ Existe una tendencia en todos los sectores a que las pymes repliquen comportamientos de las grandes empresas.
} 


\section{BIBLIOGRAFÍA}

Aragón Sánchez, A. y Rubio Bañón, A. [2005]: «Factores asociados con el éxito competitivo de las pyme industriales en España», Universia Business Review, 8, págs. 38-51.

Bahsani, S.; Himi, A.; Moubtakir, H. y Semma, A. [November 2011]: «Towards a pooling of ITIL V3 and COBIT», International Journal of Computer Sciences Issues, 8 (6), N2, págs. 185-191.

Bon, J. van; Polter, S.; Verheijen, T. y Pieper, M. [2008]: ISO/IEC 20000: una introducción, Van Haren Publishing.

Bon, J. van; Jong, A. de; Kolthof, A.; Pieper, M.; Tjassing, R.; Veen, A. van der y Verheijen, T. [2012]: Gestión de servicios TI basado en ITIL, vol. 3, Van Haren.

Bourque, P. y Fairley, R. E. (eds.) [2014]: Guide to the software engineering body of knowledge, version 3.0, IEEE Computer Society. Disponible en: www.swebok. org [Consultado: octubre de 2014].

Burton, T. y Sams, J. [2004]: Six Sigma for small and midsized organizations, J. Ross Publishing, Incorporated.

Cao, J. [2013]: Análisis detallado de la nueva ISO 27001:2013. Disponible en: http://sgsi-is027001. blogspot.com.es [Consultado: octubre de 2014].

Cater-Steel, A. y Tan, W. G. [2005]: «Implementation of IT infrastructure library (ITIL) in Australia: progress and success factors», 2005 IT Governance International Conference, Auckland University of Technology, págs. 39-52.

Chrisis, M. B.; Konrad, M. y Shrum, S. [2012]: CMMI para el desarrollo 1.3: guía para la integración de procesos y la mejora de productos, 3 . $^{\text {a }}$ ed., Universidad Ramón Areces.

Cuevas, G (coord.) [2003]: Gestión del proceso software, Centro de Estudios Ramón Areces, SA.

Cusumano, M. A. y Selby, R. W. [1998]: Microsoft secrets: how the world's most powerful software company creates technology, shapes markets, and manages people, Simon and Schuster.

EFQM [marzo 2016]: http://www.efqm.es/ [Consultada: 2014].

Fernández, C. M. [julio-septiembre 2012]: «La norma ISO 27001 del Sistema de Gestión de la Seguridad de la Información», Calidad, 3, págs. 40-44, Asociación Española para la Calidad (AEC).

Gorriti, M. y Álvarez, J. L. R. [2005]: «La contribución de las TIC al crecimiento económico en España y los retos del sector», Presupuesto y Gasto Público, 39, págs. 243-266.

Heschl, J. [2004]: «COBIT in relation to other international standards», Information Systems Control Journal, 4 , págs. $37-40$.

Institute of Systems Science [2013]: itSMF 2013 Global Survey on IT Service Management, itSMF International.

International Organization for Standardization [2005]: ISO 9000. Sistemas de gestión de la calidad. Fundamentos y vocabulario, ISO, Ginebra.

Ladino, M. I.; Villa, P. A.; López, A. M. [abril 2011]: «Fundamentos de la ISO 27001 y su aplicación en las empresas», Scientia et Technica, XVII (47), págs. 334-339, Universidad Tecnológica de Pereira. ISSN 0122-1701.

Lefcovich, M. [2009a]: Preguntas y respuestas sobre Seis Sigma, El Cid.

[2009b]: Seis Sigma: hacia un nuevo paradigma en gestión, El Cid.

Lepmets, M. [2010]: «Which process model practices support project success?», en A. Riel, R. O'Connor, S. Tichkiewitch y R. Messnarz (eds.), Systems, software and services process improvement, Springer Berlin Heidelberg, págs. 119-129.

Márquez Ramos, L.; Martínez Zarzoso, I.; Sanjuán Lucas, E. y Suárez Burguet, C. [2007]: «Efecto de las TIC sobre el comercio y el desarrollo económico. Análisis para el caso de España», Estudios de Economía Aplicada, 25 (1), págs. 313-340.

Mas, M. y Quesada, J. [2005]: Las nuevas tecnologías y el crecimiento económico en España, Fundación BBVA.

Ministerio de Industria de España [2013]: Retrato de las PYME 2013, Dirección General de Industria y de la Pequeña y Mediana Empresa, Subdirección General de Apoyo a la PYME. 
Muñoz Periñán, I. L. y Ulloa Villegas, G. V. [2011]: «Gobierno de TI-Estado del arte», Sistemas y Telemática, 9 (17), págs. 23-53.

Osiatis [2016]: www.osiatis.es [Consultado: 2015].

Potgieter, B. C.; Botha, J. H. y Lew, C. [July 2005]. «Evidence that use of the ITIL framework is effective», 18th Annual Conference of the National Advisory Committee on Computing Qualifications, Tauranga (NZ), págs. 160-167.

Randell, B. [September 1979]: «Software engineering in 1968», Proceedings of the 4th International Conference on Software Engineering, IEEE Press, págs. 1-10.
Rumbaugh, J.; Blaha, M.; Premerlani, W.; Hedí, F. y Lorensen, W. [1996]: Modelado y diseño orientados a objetos: metodología OMT, Prentice Hall.

Sammet, J. E. [1991]: «Some approaches to, and illustrations of, programming language history", Annals of the History of Computing, 13 (1), págs. 33-50.

Taghizadegan, S. [2006]: Essentials of lean Six Sigma, Butterworth-Heinemann.

Tansley, N. [2008]: A methodology for measuring and monitoring IT risk, doctoral dissertation.

Team, C. P. [2010a]: CMMI for services, version 1.3.

[2010b]: CMMI for acquisition, version 1.3.

Este máster oficial [60 créditos ECTS] se inicia en octubre y febrero de cada año y su duración normal es de 12 meses.

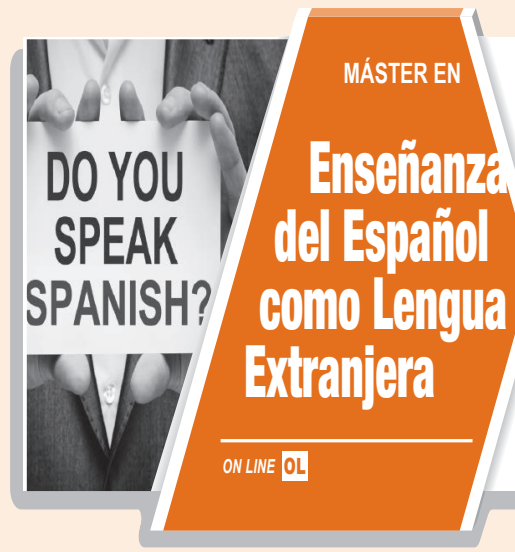

DIRIGIDO A: Titulados universitarios en las distintas áreas de Lenguas Modernas, Filología, Lingüística, Traducción, Ciencias de la Educación, Humanidades, Pedagogía y Ciencias de la Comunicación y profesionales de las lenguas que deseen especializarse en la enseñanza de español como lengua extranjera.

OBJETIVOS: Formar profesionales cualificados en el ámbito de la enseñanza del español como lengua extranjera, dotándolos de un perfil práctico y competitivo que los capacite para realizar adecuadamente y con garantía de éxito su labor profesional, facilitándoles su integración en un mercado laboral de enorme proyección internacional. 


\section{Relación de ciclos formativos de grado superior para cursar grados en la UDIMA}

La Universidad a Distancia de Madrid (UDIMA) tiene aprobado el reconocimiento de los ciclos formativos de grado superior para cursar las carreras universitarias indicadas por la Consejería de Educación de la Comunidad de Madrid:

\section{Para el Grado en Ingeniería de Organización industrial}

- Administración y Finanzas.

- Administración de Sistemas Informáticos.

- Automatización y Robótica Industrial.

- Automoción.

- Construcciones Metálicas.

- Desarrollo de Productos Electrónicos.

- Desarrollo de Proyectos Mecánicos.

- Diseño en Fabricación Mecánica.

- Instalaciones Electrotécnicas.

- Mantenimiento Aeromecánico.

- Mantenimiento de Equipo Industrial.

- Mantenimiento y Montaje de Instalaciones de Edificio y Proceso.

- Mantenimiento de Instalaciones Térmicas y de Fluidos.

- Mecatrónica Industrial.

- Industrias de Proceso Químico.

- Producción por Mecanizado.

- Programación de la Producción en Fabricación Mecánica.

- Química Industrial.

- Sistemas Electrotécnicos y Automatizados.

- Sistemas de Regulación y Control Automáticos.

- Sistemas de Telecomunicaciones e Informáticos.
Para el Grado en Ingeniería de Tecnologías y Servicios de Telecomunicación

- Administración de Sistemas Informáticos.

- Automatización y Robótica Industrial.

- Desarrollo de Aplicaciones Informáticas.

- Desarrollo de Aplicaciones Multiplataforma.

- Desarrollo de Aplicaciones Web.

- Desarrollo de Productos Electrónicos.

- Instalaciones Electrotécnicas.

- Mantenimiento Electrónico.

- Sistemas Electrotécnicos y Automatizados.

- Sistemas de Regulación y Control Automáticos.

- Sistemas de Telecomunicaciones e Informáticos

Para los Grados en Derecho o en Ciencias del Trabajo, Relaciones Laborales y Recursos Humanos

- Administración y Finanzas.

- Asistencia a la Dirección.

- Secretariado. 


\section{para cursar grados en la UDIMA}

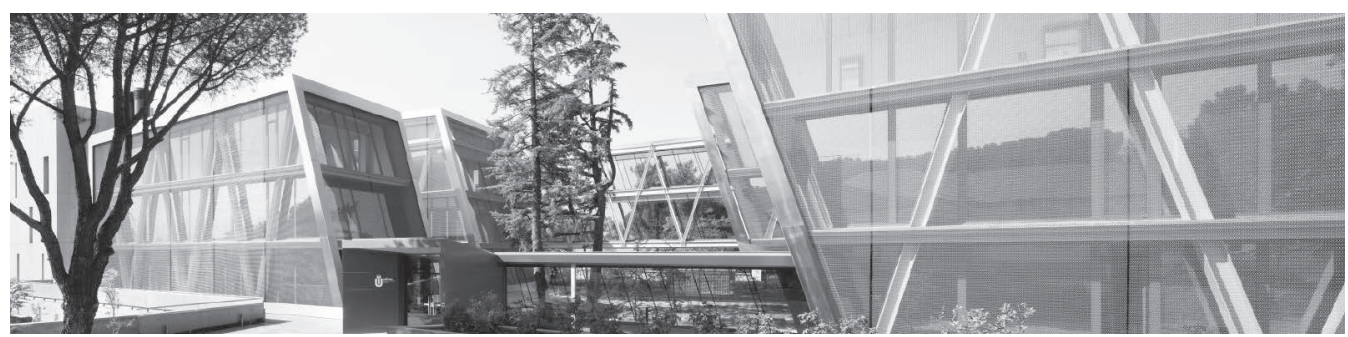

\section{< / Para el Grado en Ingeniería Informática}

- Administración de Sistemas Informáticos.

- Automatización y Robótica Industrial.

- Desarrollo de Aplicaciones Informáticas.

- Desarrollo de Aplicaciones Multiplataforma.

- Desarrollo de Aplicaciones Web.

- Sistemas de Telecomunicaciones e Informáticos.

\section{Para los Grados en Administración y Dirección de Empresas o en Economía}

- Administración y Finanzas.

- Asistencia a la Dirección.

- Comercio Internacional.

- Gestión Comercial y Marketing.

- Marketing y Publicidad.

- Secretariado.

- Transporte y Logística.

\section{Para el Grado en Marketing}

- Administración y Finanzas.

- Comercio Internacional.
- Gestión Comercial y Marketing.

- Gestión de Alojamientos Turísticos.

- Gestión de Ventas y Espacios Comerciales.

- Marketing y Publicidad.

- Transporte y Logística.

Para los Grados en Magisterio de Educación Infantil y de Educación Primaria

- Animación de Actividades Físicas y Deportivas.

- Educación Infantil.

- Integración Social.

- Animación Sociocultural y Turística.

\section{Para el Grado en Empresas y} Actividades Turísticas

- Gestión Comercial y Marketing.

- Gestión de Ventas y Espacios Comerciales.

- Gestión de Alojamientos Turísticos.

- Agencias de Viajes y Gestión de Eventos.

- Guía, Información y Asistencias Turísticas. 


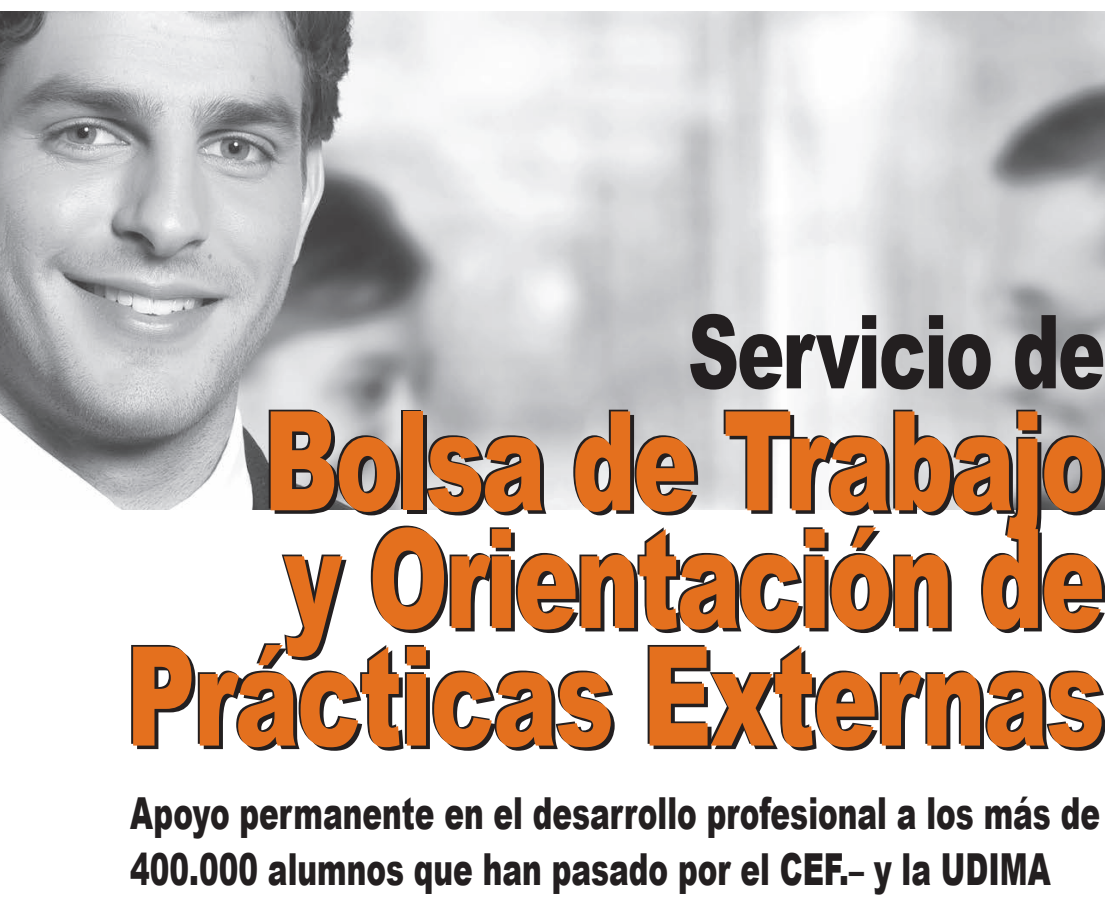

El EEF.- y la UDIMA ponen a disposición de los estudiantes diversos servicios de asesoramiento y formación para apoyar al alumno en su desarrollo académico y profesional. Es muy importante que el estudiante conozca la realidad laboral de su grado o máster, por lo que para finalizar sus estudios universitarios resulta imprescindible la realización de prácticas externas. Este servicio de Bolsa de Trabajo y Orientación de Prácticas Externas ofrece a las empresas y entidades educativas las herramientas necesarias para satisfacer sus necesidades en los procesos de selección de profesionales cualificados.

Desde nuestros orígenes hemos tenido como lema «formación para el empleo», por ello consideramos este servicio como un pilar fundamental para nuestras acciones formativas, pues todas van dirigidas a buscar empleo para nuestros alumnos, la mejora del que ya tienen o a conseguir su consolidación.

Desde el Servicio de Bolsa de Trabajo y Orientación de Prácticas Externas agradecemos tanto a las empresas y entidades educativas como a los alumnos y antiguos alumnos que utilicen este servicio como fuente de reclutamiento para los procesos de selección en todas las áreas que se imparten en el EF.- y en la UDIMA.

Este servicio es GRATUITO tanto para los estudiantes como para las entidades educativas.

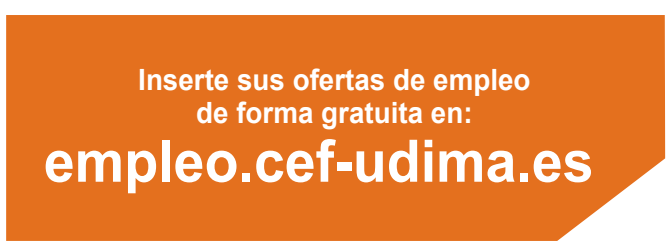

